\title{
Panorama das ações de controle da deficiência de vitamina A no Brasil
}

\section{Overview of actions to control vitamin A deficiency of in Brazil}

\author{
Maisa Cruz MARTINS ${ }^{1}$ \\ Yedda Paschoal de OLIVEIRA ${ }^{2}$ \\ Denise Costa COITINHO ${ }^{3,4}$ \\ Leonor Maria Pacheco SANTOS
}

RES U M O

\section{Objetivo}

Este trabalho propõe-se a descrever a trajetória da implantação das ações de controle da deficiência de vitamina A no Brasil.

\section{Métodos}

Trata-se de estudo baseado em dados secundários, obtidos nos registros, documentos técnicos e relatórios oficiais do Instituto Nacional de Alimentação e Nutrição, autarquia do M inistério da Saúde extinta em 1997, da Coordenação Geral da Política Nacional de Alimentação e Nutrição do M inistério da Saúde, e informações obtidas com informantes-chaves, empregando a abordagem multidimensional alicerçada na tríade: estrutura-processo-resultado.

\section{Resultados}

O Brasil foi pioneiro na iniciativa de introduzir a distribuição de vitamina A nas campanhas nacionais de imunização, estratégia posteriormente preconizada pela Organização Mundial da Saúde e pelo Fundo das Nações Unidas para a Infância. Desde 1983, o Ministério da Saúde utiliza megadoses de vitamina A, como uma das ações para combater a deficiência deste micronutriente. 0 percentual de cobertura da população alvo (crianças de 6 a 59 meses) tem aumentado, alcançando 72\% e 68\%, nos anos de 2002 e 2003, respectivamente. Essas coberturas são semelhantes às registradas nos 40 países que adotam esta estratégia. Desde 20010 programa foi ampliado para beneficiar puérperas, no pós-parto imediato.

\section{Conclusão}

Foram modestos os avanços obtidos até o ano de 1992, fato que pode ser explicado pela falta de regularidade na distribuição das cápsulas de vitamina A. A partir de 1994, o programa manteve certa constância, porém

\footnotetext{
${ }^{1}$ Ministério da Saúde, Agência Nacional de Saúde Suplementar, Gerência de Integração com o Sistema Único de Saude. Rio de Janeiro, RJ, Brasil.

2 Ministério da Saúde. Coordenação Geral da Política de Alimentação e Nutrição. Braślilia, DF, Brasil.

3 Universidade de Brasília, Faculdade de Saúde, Departamento de N utrição. Campus Universitário Darcy Ribeiro, 70910-900, Brasilia, DF, Brasil. Correspondência para/Correspondence to: L.M.P. SANTOS. E-mail: বeopac@unb.br>.

${ }^{4}$ Organização Mundial da Saúde, Unidade de Nutrição para Saúde e Desenvolvimento. Genebra, Suíça.
} 
persiste a necessidade de promover ações mais consolidadas nos serviços de saúde com maior alcance social, para além da distribuição rotineira dessas cápsulas.

Termos de indexação: deficiência de vitamina A; prevenção \& controle; Brasil.

\section{A B S T R A C T}

\section{Objective}

This manuscript aims to describe the implementation of actions to control vitamin A deficiency in Brazil.

\section{Methods}

The study was based on secondary data obtained from official reports and technical documents from the former Instituto Nacional de Alimentação e Nutrição (inactivated in 1997), from the Coordenação Geral da Política Nacional de Alimentação e Nutrição, M inistry of Health and information obtained from key informants employing the multidimensional approach based on the triad: structure-process-result.

\section{Results}

Brazil was pioneer in taking the initiative of linking vitamin A distribution to the National Immunization Days, a strategy that later on was recommended by the World Health Organization and the United Nations Children's Fund. Since 1983, vitamin A megadoses are employed as a strategy to fight this micronutrient deficiency by the Ministério da Saúde. Coverage of the target population (children 6-59 months old) is steadily increasing, reaching $72 \%$ and $68 \%$ in the years 2002 and 2003 respectively. These coverage rates are similar to those reported in the 40 countries that have also adopted this strategy. Since 2001 the program was extended to lactating women, in the immediate post-partum period.

\section{Conclusion}

The program achievements until 1992 were very modest, fact that could be explained by the irregularity of vitamin A capsule distribution. From 1994 on the distribution was more constant, but the need to promote more integrated actions in the health services, as well as far reaching social actions, beyond the routine distribution of vitamin A capsules, still remains.

Indexing terms: vitamin A deficiency; prevention \& control; Brazil.

\section{N T RO D U ÇÃ O}

Os distúrbios do estado nutricional durante a infância podem ter causalidade múltipla. Predominam nos países em desenvolvimento, as deficiências alimentares - em especial de micronutrientes - que são fatores de risco para a saúde e sobrevivência de grupos vulneráveis, como crianças em idade pré-escolar.

Dentre as deficiências nutricionais de maior importância epidemiológica, a deficiência de vitamina $\mathrm{A}$ ainda hoje assume graves proporções no contexto da saúde pública em todo o mundo. A deficiência prolongada dessa vitamina causa alterações no revestimento ocular, levando a um quadro de cegueira irreversível (xeroftalmia e ceratomalácia). Além disso, a vitamina A encon- tra-se associada à integridade epitelial e às funções imunes, sendo sua deficiência responsável por um aumento no risco de morbidade e mortalidade na infância1-5.

O Estudo Nacional da Despesa Familiar (ENDEF), realizado entre 1974 e 1975, pelo Instituto Brasileiro de Geografia e Estatística (IBGE), demonstrou que o déficit dessa vitamina era a carência alimentar de maior magnitude e abrangência na dieta brasileira nos anos $70^{6}$. Estudos realizados em Pernambuco ${ }^{7-9}$, Ceará ${ }^{9}$, Rio Grande do Norte ${ }^{10}$, Paraíba ${ }^{8,9,11,12}$ e Bahia ${ }^{13}$ confirmaram, desde a década dos 80 a necessidade de combater a deficiência de vitamina $A$ em crianças de 0 a 5 anos nos estados da Região Nordeste. Essa deficiência também foi encontrada em pré-escolares de 0 a 72 meses, no vale do Jequitinhonha, em Minas Gerais ${ }^{14}$. 
Quanto aos indicadores bioquímicos de deficiência de vitamina A, a Organização M undial da Saúde (OMS) recomenda que níveis de retinol sérico menores que $0,70 \mathrm{micromol} /$ litro são indicativos de hipovitaminose $A$ grave, enquanto que os acima de 1,75 micromol/litro, demonstram níveis seguros e adequados de reserva orgânica dessa vitamina ${ }^{2}$. A OMS sugere ainda a seguinte interpretação para a prevalência de baixos níveis de retinol sérico: entre 2,0\% e 9,9\% - Problema de Saúde Pública Leve; entre 10,0 e 19,9\% - Problema de Saúde Pública Moderado; - $\geq 20,0 \%$ - Problema de Saúde Pública Grave

Estudo com amostra representativa, realizado no estado da Paraíba ${ }^{15}$, em 1992, demonstrou que $16,0 \%$ das crianças menores de cinco anos apresentavam a hipovitaminose $A$, indicando um problema de Saúde Pública moderado. Outros estudos, também com amostras de base populacional, realizados nos estados de Pernambuco, em 199716, e Sergipe, em 199817, constataram baixos níveis de retinol em 19,3\% e $32,1 \%$ dos menores de cinco anos, respectivamente, confirmando a deficiência de vitamina $A$ como importante problema de saúde pública no nordeste brasileiro. Ainda outros estudos, recentes, em 3 capitais da Amazônia Ocidental (Boa Vista, Manaus e Porto Velho), encontraram baixos níveis de retinol em $15,5 \%, 19,6 \%$ e $32,4 \%$ dos pré-escolares, respectivamente ${ }^{18}$.

A administração de megadoses de vitamina A a crianças nessa faixa etária, constitui-se em uma das principais intervenções, a curto prazo, para combater a deficiência dessa vitamina em nível de saúde pública. A suplementação com vitamina $A$ em regiões onde a deficiência é endêmica, favorece ainda a redução da mortalidade nas crianças menores de cinco anos de idade entre $23 \%$ e $30 \%{ }^{4,19}$, e representa, também, a possibilidade de atenuar a severidade das doenças diarréicas ${ }^{4,20}$.

\section{M ÉTODOS}

Trata-se de estudo baseado em dados secundários, referentes ao período de 1983 a
2003, diretamente de registros, documentos técnicos e relatórios oficiais do Instituto Nacional de Alimentação e Nutrição (INAN) (autarquia do Ministério da Saúde extinta em 1997), como também em planilhas de monitoramento do Programa Nacional de Controle da Deficiência de Vitamina A e da Coordenação Geral da Política Nacional de Alimentação e Nutrição (CGPAN), do Ministério da Saúde. Além dessas, algumas informações foram obtidas por meio de entrevistas com informantes-chaves: do is gestores nacionais e três pesquisadores, todos com longa experiência no programa.

Para uma melhor sistematização dos conteúdos e apresentação desta análise, foi adotado o enfoque multidimensional, alicerçado na tríade: estrutura-processo-resultado, descrito por diversos autores $^{21-23}$ e adaptado para o caso de políticas de alimentação e nutrição por um dos autores deste estudo ${ }^{24}$. Na dimensão estrutura, foram consideradas as características relativamente estáveis, como instrumentos normativos, competências organizacionais e infra-estrutura para monitoramento e controle do programa. Já na dimensão de processo, levaram-se em conta atividades e procedimentos relacionados às estratégias de provimento e distribuição das megadoses de vitamina $A$, fluxo de informações, bem como 0 monitoramento do programa. Por fim, a dimensão resultado foi representada pela cobertura do Programa nas áreas de risco e na população alvo.

\section{RESULTA DOS E DISCUSSÃ O}

\section{Histórico do controle da deficiência de vitamina A no Brasil - período 1983-1993}

A primeira proposta brasileira de ação específica para o combate da deficiência de vitamina A ocorreu em 1979, quando foi elaborado um projeto de intervenção semestral, com doses maciças de vitamina e fortificação de açúcar, na região nordeste. Porém esse projeto não foi executado por insuficiência de dados epide- 
miológicos que justificassem medidas de grande abrangência ${ }^{25}$.

Foi a partir de 1983 que as atividades de controle da deficiência de vitamina A tiveram início no Brasil, ainda que de modo incipiente. A estratégia adotada foi a de suplementação com megadoses de vitamina $A$, para crianças préescolares, em áreas específicas de alto risco de desenvolvimento da deficiência desse micronutriente, por suas características geográficas, climáticas e econômicas. As doses empregadas eram de 100.000UI (Unidades Internacionais) para crianças de 6 a 11 meses, e de $200.000 \mathrm{UI}$, para as de 12 a 59 meses de idade, tendo como esquema operacional básico a sua distribuição nos Dias Nacionais de Vacinação.

O Brasil foi o pioneiro na estratégia de distribuição de vitamina $\mathrm{A}$ integrada ao programa nacional de imunização ${ }^{13,14}$. Posteriormente, essa iniciativa foi preconizada pela Organização Mundial de Saúde (OMS), United Nations Children's Fund (UNICEF) e International Vitamin A Consultative Group (IVACG), como forma de tornar mais eficiente o combate dessa deficiência a curto prazo ${ }^{26}$.

Durante uma década, a distribuição de megadoses de vitamina A ocorreu de forma descontínua, com intervenções mais constantes nos estados da Paraíba e Pernambuco, mas com grandes flutuações na cobertura. M esmo assim, em alguns perío dos, a distribuição foi expressiva, atingindo mais de 1 milhão de crianças em 1986 e em 1991 (Tabela 1).

\section{Estrutura do programa}

A estrutura normativa do programa surgiu em 1994, quando o M inistério da Saúde criou o Programa Nacional de Controle das Deficiências de Vitamina A, no âmbito do INAN, por meio da Portaria n.2.160, de 29 de Dezembro de $1994^{27}$ - o marco legal que permitiu a expansão do programa. A partir da data de publicação da Portaria, as megadoses de vitamina A continuaram provenientes de doações internacionais, contudo, com maior regularidade e participação de maior número de estados. A partir de então, 0 número de beneficiários situou-se acima do patamar de 1,5 milhões (Figura 1 e Tabela 2).

Em 1997, ocorreu o processo de extinção do INAN, acarretando a revogação da Portaria 2.160 e a paralisação quase total do programa. Em 1999, houve uma retomada do programa pela Área Técnica de Alimentação e Nutrição do M inistério da Saúde, que atualmente é de responsabilidade direta da Coordenação Geral da Política

Tabela 1. Distribuição nacional de megadoses de vitamina A para crianças de 6 a 59 meses por unidade da Federação, no período anterior à Portaria no 2.160 que instituiu o Programa Nacional de Vitamina A. Brasil, 1983-1992.

\begin{tabular}{|c|c|c|c|c|c|c|c|c|c|}
\hline \multirow{2}{*}{ Ano } & \multicolumn{9}{|c|}{ Número de megadoses de vitamina A distribuídas por Unidade da Federação } \\
\hline & PB & PE & RN & $\mathrm{AL}$ & MG & CE & PI & SE & Total \\
\hline 1983 & 100.420 & 135.000 & - & - & 149.000 & - & - & - & 384.420 \\
\hline 1984 & 183.672 & 222.782 & - & - & 140.000 & - & - & - & 546.454 \\
\hline 1985 & 133.224 & - & - & - & - & - & - & - & 133.224 \\
\hline 1986 & - & 105.753 & 149.083 & 381.909 & - & - & 279.357 & 169.000 & 1.085 .102 \\
\hline 1987 & 299.739 & 300.000 & - & - & - & - & - & - & 599.739 \\
\hline 1988 & 487.026 & - & - & - & - & - & - & - & 487.026 \\
\hline 1989 & 105.300 & 5.000 & 500 & - & - & - & - & - & 110.800 \\
\hline 1990 & 279.317 & - & 500 & - & - & - & - & - & 279.817 \\
\hline 1991 & 329.948 & - & 191.000 & 337.000 & - & 863.000 & - & - & 1.720 .948 \\
\hline 1992 & 265.186 & - & 255.567 & 228.363 & - & - & - & - & 749.116 \\
\hline
\end{tabular}

Fonte: (1983-1992) - Dados internos do Instituto Nacional de Alimentação e Nutrição, Coordenação Geral da Política de Alimentação e Nutrição (INAN/CGPAN); (1993) Dados não disponíveis.

PB: Paraíba; PE: Pernambuco; RN: Rio Grande do Norte; AL: Alagoas; MG: Minas Gerais; CE Ceará; PI: Piauí; SE: Sergipe. 
de Alimentação e Nutrição (CGPAN), que hierarquicamente está subordinada ao Departamento de Atenção Básica (DAB) da Secretaria de Atenção à Saúde (SAS), do M inistério da Saúde. É executado por intermédio das Secretarias Estaduais e Municipais de Saúde, envolvendo as coordenações estaduais de alimentação e nutrição. Também conta com o apoio UNICEF, Organização Pan-Americana da Saúde (OPAS) e colabo- ração técnica dos Centros Colaboradores em Alimentação e Nutrição, do M inistério da Saúde.

Recentemente, o Ministério da Saúde publicou a portaria 729 , de 13 de maio de 2005, instituindo novamente o Programa Nacional de Suplementação de Vitamina $A^{28}$, honrando 0 compromisso assumido, junto às Nações Unidas, de controlar a deficiência dessa vitamina e suas conseqüências. Entre outras atribuições, compete

Tabela 2. Distribuição nacional de megadoses de vitamina A para crianças de 6 a 59 meses por unidade da Federação a partir da publicação da Portaria № 2.160 que instituiu o Programa Nacional de Vitamina A. Brasil, 1994-2003.

\begin{tabular}{cccccccccccccc}
\hline \multirow{2}{*}{ Ano } & \multicolumn{10}{c}{ Número de megadoses de vitamina A distribuídas por Unidade da Federação } \\
\cline { 2 - 12 } & PB & PE & RN & AL & M & CE & PI & SE & M A & BA & Total \\
\hline 1994 & 98.350 & 445.235 & 200.394 & 116.056 & 71.459 & 515.005 & 258.403 & 112.879 & 406.939 & - & 2.224 .720 \\
1995 & 290.398 & 214.063 & 199.734 & - & 47.230 & 195.444 & 192.356 & 42.696 & 166.031 & 202.280 & 1.553 .232 \\
1996 & 295.447 & 249.617 & 158.777 & - & 43.762 & - & 231.894 & 54.464 & 207.572 & 313.304 & 1.554 .837 \\
1997 & ND & ND & ND & ND & ND & ND & ND & ND & ND & ND & ND \\
1998 & ND & ND & ND & ND & ND & ND & ND & ND & ND & ND & 1.864 .379 \\
1999 & 237.639 & 362.724 & 160.029 & 337.206 & 74.624 & 309.910 & 36.078 & 117.615 & 142.407 & 701.511 & 2.479 .743 \\
2000 & 269.593 & 272.400 & 56.452 & 153.251 & 62.267 & 636.955 & 31.661 & 43.737 & 243.665 & 414.946 & 2.184 .927 \\
2001 & 117.807 & 402.719 & 23.643 & 164.992 & 175.761 & 751.006 & 61.900 & 101.036 & 118.590 & 212.517 & 2.129 .971 \\
2002 & 256.854 & 607.221 & 52.633 & 160.226 & 569.586 & 1.065 .013 & 82.573 & 134.467 & 269.793 & 352.642 & 3.551 .008 \\
2003 & 275.667 & 531.725 & 113.358 & 155.372 & 390.687 & 718.677 & 131.011 & 74.300 & 608.039 & 351.813 & 3.350 .649 \\
\hline
\end{tabular}

Fontes: (1997) Dados não disponíveis; (1994 - 2002) Dados disponíveis no site www.saude.gov.br/nutricao; (1998) Dados não disponíveis por estado. Goodman34; (2003) Dados internos da Coordenação Geral da Política de Alimentação e Nutrição (CGPAN).

PB: Paraíba; PE: Pernambuco; RN: Rio Grande do Norte; AL: Alagoas; M G: Minas Gerais; CE Ceará; PI: Piauí; SE: Sergipe; MA: Manaus; BA: Bahia.

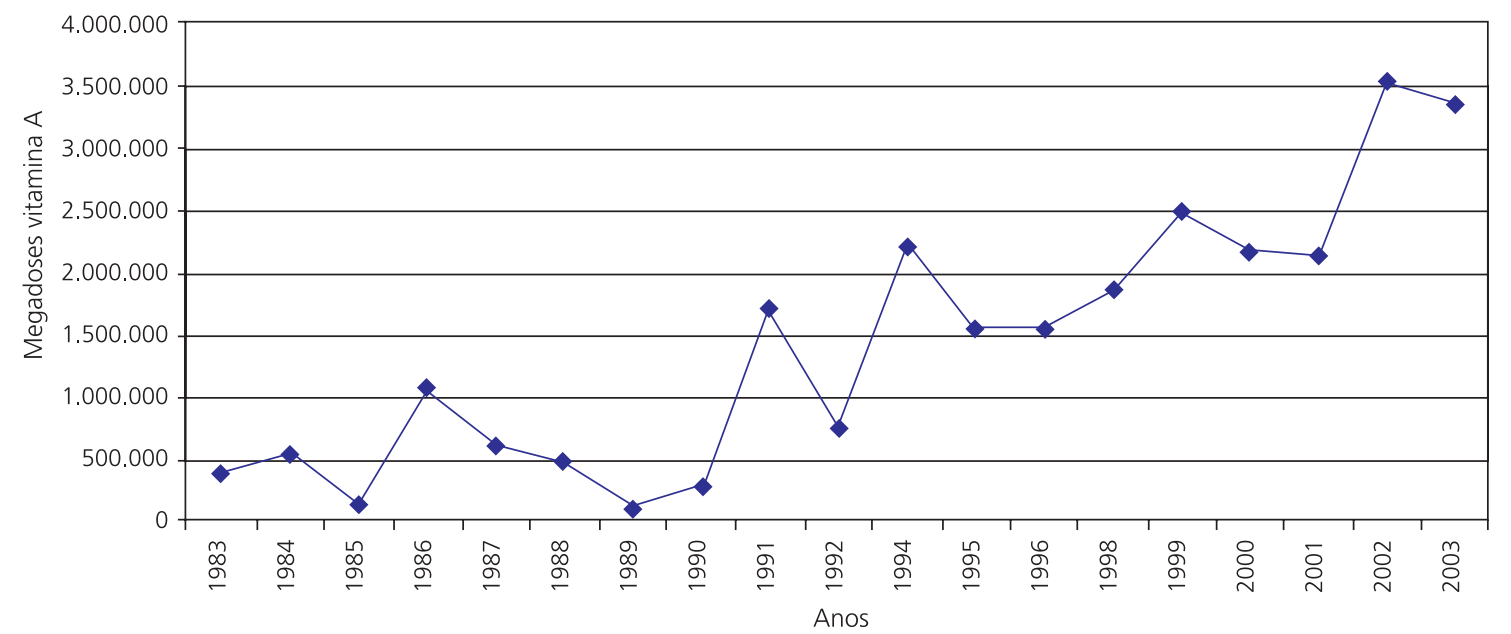

Figura 1. Evolução da distribuição nacional de megadoses de vitamina A para crianças de 6 a 59 meses. Brasil, $1983-2003$.

Fontes: (1983-1992) Dados internos da (Coordenação Geral da Política de Alimentação e Nutrição (CGPAN); (1993 e 1997) Dados não disponíveis; (1994-2002) Dados disponíveis no site www.saude.gov.br/nutricao; (1998) Goodman34; (2003) Dados internos da Coordenação Geral da Política de Alimentação e Nutrição (CGPAN). 
ao M inistério da Saúde a aquisição e o envio do suplemento de vitamina $A$; 0 acompanhamento da implantação e o monitoramento da cobertura populacional; a avaliação do desempenho e o impacto do Programa no plano nacional, e o apoio das ações da mesma natureza nos estados e municípios; o fomento de atividades complementares de promoção da alimentação saudável e prevenção de deficiência de vitamina $\mathrm{A}$ e a elaboração de materiais e divulgação das normas operacionais do Programa aos estados.

\section{Processos do programa}

Durante uma década (1983-1994), a obtenção do suplemento ocorreu por meio de doações de cápsulas por instituições internacionais, como UNICEF, Sight and Life e Cruz Vermelha (esta última no Ceará). 0 processo de extinção do INAN, entre 1997 e 1998, levou ao agravamento de problemas logísticos já existentes, destacando-se, dentre eles, a suspensão da aquisição das cápsulas de megadoses via UNICEF.

Mas, a partir de 1999, o programa foi retomado e nessa ocasião as megadoses de vitamina $A$ passaram a ser fornecidas pela instituição M icronutrients Iniciative (M I), do Canadá, com a interveniência da Organização Panamericana de Saúde. Ainda persistia a irregularidade nas entregas dessas megadoses, o que provocava grande atraso na chegada aos municípios. To do 0 estoque de vitamina $A$ era centralizado no porto ou no aeroporto da cidade de Fortaleza, CE, surgindo, quase sempre, dificuldade nos procedimentos alfandegários para a liberação do produto, apesar de se tratar de uma doação de medicamentos. Não foi possível obter registros quantitativos sobre as doações nesse período, tendo em vista que a documentação referente a esse processo foi arquivada durante a extinção do INAN.

A partir de 2001, visando ao aperfeiçoamento na gestão e monitoramento do programa, o M inistério da Saúde passou a fazer a aquisição direta das cápsulas de vitamina $\mathrm{A}$, por intermédio da FarManguinhos (Fundação Oswaldo Cruz), localizada no Rio de Janeiro. Nesse ano foram adquiridas e encaminhadas às Secretarias Estaduais de Saúde 5,5 milhões de cápsulas de megadoses de vitamina A (600 mil cápsulas de 100.000 U e 4,9 milhões de cápsulas de 200.000 UI); em 2002, foram 6,8 milhões de cápsulas (800 mil de 100.000 Ul e 6 milhões de 200.000 UI), e em 2003, 10 milhões de cápsulas (1,2 milhões, de 100.000 Ul e 8 milhões de 200.000 UI). As quantidades de megadoses de vitamina $A$ adquiridas por parte do M inistério da Saúde foram suficientes para atender toda a população-alvo do Programa (crianças de 6 a 59 meses de idade), nas regiões consideradas de risco epidemiológico de deficiência dessa vitamina (Nordeste e Vale do Jequitinhonha, MG), considerando-se uma megadose por criança.

Porém, levando-se em consideração a recomendação da OMS de que cada criança deveria receber megadoses de vitamina $A$, em intervalos de quatro a seis meses, a quantidade real de megadoses de 200.000 U necessária para atender $100 \%$ das crianças de 12 a 59 meses, deveria ser multiplicada por dois em cada ano: assim, seriam 8,6 milhões em 2001; 8,7 milhões em 2002 e 8,7 milhões em 2003. A área técnica, responsável pelo cálculo da quantidade de cápsulas a serem adquiridas pelo Ministério da Saúde, no entanto, justifica que foram levados em consideração os estoques anteriores existentes nos estados, que somados ao adquirido pelo Ministério da Saúde, seriam suficientes para atender $100 \%$ do público-alvo, considerando duas doses/ano. E que, ainda, a quantidade de cápsulas de 100.000 U I adquiridas pelo M inistério da Saúde foi suficiente para atender a toda a população-alvo (crianças de 6 a 11 meses) nos anos de 2001 a 2003.

A Portaria 729, de 2005, detalhou as competências e os procedimentos do programa. Cabe às Secretarias Estaduais de Saúde o processo de distribuição das megadoses aos municípios, 0 monitoramento local do Programa, o fornecimento de relatórios periódicos ao M inistério da Saúde, 
bem como a indicação de área técnica responsável para coordenar, em âmbito estadual, a operacionalização do Programa. Essa esfera de governo deve ainda: garantir a divulgação da norma operacional do Programa aos municípios, bem como apoiá-los na implantação dessas condições; assessorar os municípios na identificação da estratégia de ação para suplementação das crianças entre 6 e 59 meses de idade, de forma periódica e regular; apoiar a capacitação de recursos humanos nas ações de saúde inerentes ao controle e à prevenção das carências nutricionais, com ênfase na promoção da alimentação saudável; avaliar o desempenho e o impacto estadual do Programa e apoiar os municípios para o desenvolvimento dessas ações.

A distribuição das megadoses para 0 público-alvo cabe aos gestores municipais, de acordo com o processo de descentralização previsto pelo Sistema Único de Saúde (SUS). As estratégias de distribuição comumente empregadas pelos municípios são as Campanhas de Multivacinação e a associação com a rotina de vacinação ou do atendimento ambulatorial de pediatria nas Unidades de Saúde, com os Agentes Comunitários.

Segundo a nova Portaria, são condições necessárias à implantação do Programa, a serem observadas pelos municípios: o recebimento e 0 armazenamento da megadose de vitamina $\mathrm{A}$ em local apropriado, bem como a utilização, em tempo hábil, de acordo com o prazo de validade; a identificação das famílias que tenham crianças de 6 a 59 meses e a administração da megadose dessa vitamina; a implantação e a implementação do Programa Nacional de Suplementação de Vitamina A em todas as maternidades municipais; o provimento das ações básicas de saúde e atividades educativas em alimentação e nutrição, necessárias para que as famílias reconheçam a deficiência de vitamina A como problema de saúde, e adotem hábitos alimentares saudáveis; a manutenção atualizada dos dados de controle do estoque e de distribuição das megadoses, enviando mensalmente as informações para os níveis federal e estadual; e a capacitação de profissionais de saúde para operacionalização do Programa.

\section{Resultados do programa - período 1994-2003}

O acompanhamento da suplementação é feito pelo controle do número de doses distribuídas, não havendo, até o momento, um controle do número real de crianças suplementadas, visto que uma criança na faixa etária de 12 a 59 meses, teoricamente, deveria receber duas doses/ano. Para as crianças de 6 a 11 meses, esse problema não tem grande impacto, pois nessa faixa etária, provavelmente, a criança só fará uso de uma dose/ano. Logo, para efeito de análise dos dados desta publicação, será considerado que para cada dose distribuída, obtém-se uma criança beneficiada. Vale ressaltar que ainda persistem falhas no sistema de distribuição de megadoses de vitamina A no Brasil.

Não será analisada a cobertura da distribuição das megadoses de vitamina $\mathrm{A}$ anterior ao ano de 1994, visto que nesse período esse processo ocorria de forma isolada e esporádica por alguns estados.

A pós a instituição da portaria que regulamentou o programa, em 1994, a cobertura oscilou entre $28 \%$ e $72 \%$ (Figura 2). Observa-se que no ano da implantação oficial do Programa, houve uma cobertura superior a 40\%, o que não ocorreu nos dois anos subseqüentes (1995 e 1996). Provavelmente, os estados ainda não haviam estabelecido uma rotina de distribuição das megadoses, necessitando de tempo para sistematizar o processo nas unidades de saúde.

Durante o período de 1997 a 1998, não houve nova doação de megadoses de vitamina A, mas os estados ainda utilizaram o saldo de cápsulas dos anos anteriores. Não foi possível localizar os dados referentes a 1997, em função da extinção do INAN, mencionada anteriormente. A partir de 1999, o processo de distribuição das 


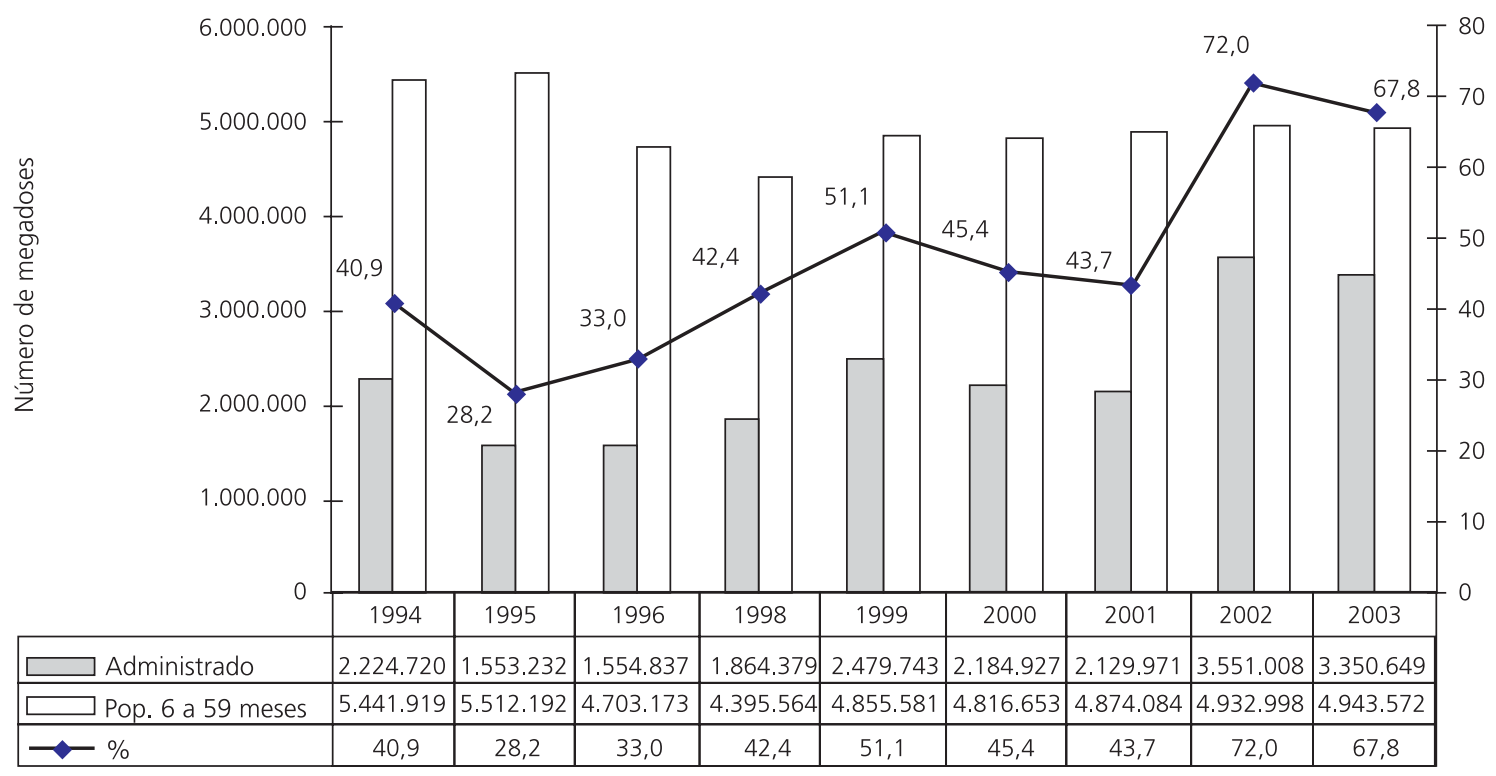

Figura 2. Cobertura e doses de vitamina A distribuídas para crianças de 6 a 59 meses. Brasil, 1994 a 2003.

megadoses foi intensificado, sendo administradas mais de 2 milhões de megadoses por ano, sendo o programa novamente baseado em doações internacionais. A partir de 2001, o Ministério da Saúde passou a adquirir diretamente as cápsulas gelatinosas de vitamina A e foram administrados 2,1 milhões de doses. Os dados de 2002 e 2003 revelaram que foram administrados mais de 3 milhões de doses para crianças de 6 a 59 meses de idade (cobertura de $72,0 \%$ e $67,8 \%$, respectivamente) (Figura 2).

Observa-se que os dados apresentados para a cobertura da distribuição de megadoses de vitamina A em crianças de 6 a 11 meses são, geralmente, mais elevados do que os dados apresentados para crianças de 12 a 59 meses de idade (Figura 3). Essa cobertura mais elevada deve-se, provavelmente, aos contatos rotineiros com os serviços primários de atenção à saúde, como a imunização, para essa faixa etária.

Em 2001, além dos municípios da região Nordeste e dos municípios do Vale do Jequitinhonha, em Minas Gerais, o programa foi ampliado para três municípios no Estado de São Paulo (Nova Odessa, Hortolândia e Sumaré).

Também em 2001, o programa foi ampliado para atendimento a puérperas, no pós-parto

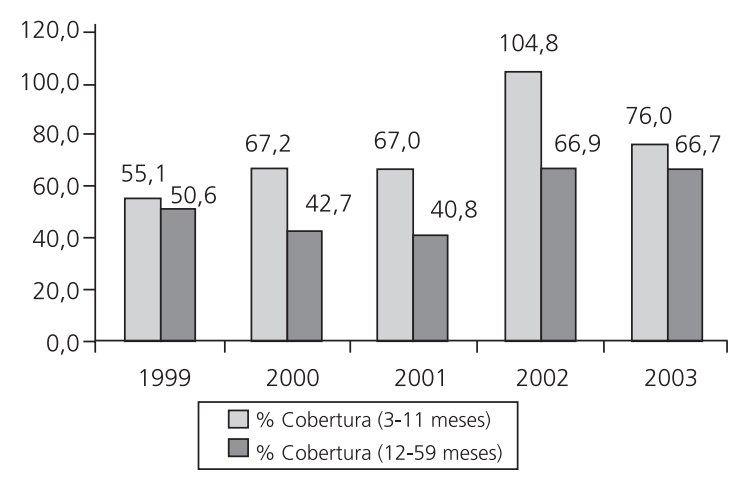

Figura 3. Cobertura da distribuição de vitamina A para crianças por faixa etária. Brasil, 1999-2003.

imediato, visando a garantir a adequação das reservas corporais maternas, por meio da suplementação com 200.000UI nas maternidades e/ou hospitais. Dessa forma, o aporte de vitamina A, do leite materno, para as crianças menores de 6 meses amamentadas, encontra-se garantido. Neste ano de 2001, a estratégia foi adotada por seis estados (Alagoas, M aranhão, M inas Gerais, Paraíba, Piauí e Rio Grande do Norte) e pelo Instituto $M$ aterno Infantil (IM IP), em Recife, PE, sendo suplementadas um total de 46.380 puérperas. Em 2002, a cobertura foi ampliada para outros três estados (Bahia, Ceará e Pernambuco), sendo 
alcançadas 59.664 puérperas; já em 2003, foram suplementadas 76.671 mulheres. Ressalta-se que o aleitamento materno é o grande fator protetor da deficiência de vitamina $A$ até os dois anos de idade, fase de maior vulnerabilidade.

\section{Avaliações do Programa Nacional de Controle da Deficiência de Vitamina A}

Não existe ainda uma sistemática de avaliação para o Programa. Em estudo longitudinal, realizado em 1985, para avaliar a eficácia da campanha de distribuição de megadoses de vitamina $A$, no estado da Paraíba ${ }^{29}$, concluiu-se que esse tipo de intervenção era eficaz. Na amostra de 120 crianças acompanhadas, 2,5\% apresentavam níveis séricos de retinol abaixo de 0,70 micromol/litro e $16,6 \%$ entre 0,70 e 1,05 micromol/litro antes da suplementação. Trinta dias após receber o suplemento, distribuído no Dia Nacional de Vacinação, nenhuma criança permaneceu com níveis abaixo desses limites; 37,3\% passaram a ter níveis entre 1,05 e 1,75 micromol/ litro e $62,5 \%$, acima de 1,75 micromol/litro. 0 estudo concluiu que essa era uma estratégia rápida, eficaz e de baixo custo para combater a deficiência dessa vitamina.

Outro estudo, controlado, envolvendo 852 crianças, no estado da Bahia, foi realizado com o objetivo de identificar a ocorrência e a natureza de possíveis efeitos adversos agudos, em conseqüência da suplementação com megadoses de vitamina A oferecida junto com a imunização em massa. Os resultados demonstraram que em ambos os grupos (suplementado e controle) houve uma incidência igual (e muito baixa) de diarréia, febre, vômito e anorexia, sugerindo que não existem efeitos adversos quando a vitamina $A$ é oferecida junto com as vacinas antipólio oral, DPT e anti-sarampo ${ }^{30}$.

A mais extensa avaliação do programa, do ponto de vista da estrutura, processos e resultados, foi realizada entre 1995 e 1998, em 44 municípios do estado da Bahia, e envolveu 2.546 crianças de 0 a 5 anos, provenientes de 1.344 famílias de baixa renda ${ }^{31}$ e concluiu:
- O arcabouço legal do Programa Nacional de Controle das Deficiências de Vitamina A (PNVITA), que envolve a Portaria 2.160 do Ministério da Saúde, na qual foram explicitados claramente os seus objetivos e estratégias, foi considerado adequado, muito embora a referida portaria não estivesse vigente à época do estudo;

- Em termos de processos (aquisição das cápsulas de vitamina $A$, a produção de materiais informativos e educativos, a realização do treinamento e o monitoramento das áreas de risco), observou-se que o processo de extinção do INAN repercutiu negativamente na implementação do Programa;

- Mesmo considerando os biênios 19951996 e 1997-1998 como fases iniciais de implementação do PNVITA na Bahia, foram modestos os resultados obtidos nesse período. Observou-se baixa cobertura da suplementação com megadoses de vitamina A, pois apenas $37,5 \%$ das crianças de 6 a 59 meses receberam pelo menos uma dose no período do estudo. Foi observado que mais de $90,0 \%$ das crianças receberam apenas uma dose ao ano. E foi notória a falta de conhecimentos sobre vitamina A por parte dos entrevistados. Destes, $74,6 \%$ referiram não saber absolutamente nada sobre essa vitamina, e apenas $10,9 \%$, tiveram acesso a algum tipo de informação, acarretando uma falta de conscientização das famílias sobre os benefícios da suplementação com essa vitamina ${ }^{31}$.

Vale ressaltar que no Brasil, até a data atual, não existe um sistema nacional de vigilância epidemiológica sobre a hipovitaminose $\mathrm{A}$ e a xeroftalmia, logo, não é possível avaliar o impacto das medidas de prevenção pelo Programa Nacional de Controle da Deficiência de Vitamina A. No entanto, dois estudos de corte transversal, com amostras representativas de pré-escolares, no estado Paraíba (1982-1992), demonstraram que a xeroftalmia e a cegueira nutricional foram virtualmente eliminadas após o início da suplementação com vitamina $A$, que ocorreu em $1983^{15}$. 
Outras medidas também têm sido adotadas para o controle dessa deficiência pelo Ministério da Saúde, tais como:

- Desenvolvimento de material didático e informativo para estímulo ao consumo de alimentos, fontes de vitamina A. O Brasil é riquíssimo em alimentos regionais, fontes dessa vitamina, como o dendê, a azedinha, a bertalha, o caruru, o mamão, a manga, a pupunha, a vinagreira, a batata doce, o buriti, o caruru, o jerimum, o pequi, o ora-pro-nobis, a beldroega, a taioba, a serralha, entre outros tantos. Cabe resgatá-los e reincorporá-los à nossa cultura alimentar o quanto antes. Assim, o M inistério da Saúde publicou uma compilação dos alimentos disponíveis nas regiões brasileiras que são fonte desta e de outras vitaminas e minerais ${ }^{32}$. Este fará parte do material de trabalho das equipes de Saúde da Família e dos Agentes Comunitários de Saúde de todo o País.

- Maior envolvimento dos centros de pesquisas no diagnóstico do problema e avaliação de impacto das medidas de intervenção adotadas.

- Maior sensibilização dos gestores estaduais e municipais para a ampliação da cobertura da distribuição da megadoses de vitamina A.

Outra iniciativa do Ministério da Saúde merecedora de destaque é a recém iniciada pesquisa nacional, que permitirá o diagnóstico da hipovitaminose $\mathrm{A}$ e da anemia. 0 objetivo da pesquisa será fornecer indicadores de nutrição que subsidiem a avaliação dos avanços alcançados nessa área, e auxiliem a formulação de políticas e estratégias de ação, para promover uma redução e/ou erradicação dessa deficiência nutricional.

O Ministério da Saúde visa a dar continuidade às ações desenvolvidas e ampliar mecanismos de controle, tanto assim que publicou, recentemente, a Portaria 729 nesse sentido (28), normatizando como se deve promover a redução e/ou erradicação dessa deficiência nutricional, por meio das seguintes ações:

- dar continuidade ao processo de aquisição direta das cápsulas de vitamina $\mathrm{A}$;

- modificar o formato da cápsula, para abri-la, sem empregar tesouras;
- fornecer suporte técnico-científico sobre vitamina A para os Estados e M unicípios;

- ampliar a cobertura, com doses semestrais, do número de crianças suplementadas com vitamina $A$;

- monitorar e ampliar a implantação do programa para puérperas, nas unidades hospitalares;

- desenvolver pesquisa nacional sobre 0 diagnóstico da hipovitaminose A no Brasil, de forma a subsidiar as intervenções;

- avaliar o suporte técnico desenvolvido pelos Estados e Municípios para acompanhar o Programa: planilhas, informativos, capacitação, utilização dos meios de comunicação, disponibilidade de veículos, etc.;

- avaliar as estratégias utilizadas para administrar o suplemento de vitamina A: rotina dos serviços de saúde, campanhas de multivacinação, agentes comunitários de saúde, etc.;

- implantar um sistema informatizado para transmissão on line de informações sobre os estoques de vitamina $A$, doses administradas, perdidas e outras situações.

\section{A experiência internacional com a distribuição de vitamina A}

Conforme já descrito, o Brasil foi pioneiro na iniciativa de associar a distribuição de vitamina A aos dias nacionais de vacinação. Apesar de pouco documentada no País, a experiência brasileira foi divulgada amplamente em eventos internacionais ao longo dos anos 80 e 90 , sobretudo nas reuniões do International Vitamin A Consultative Group, organização que reúne gestores e pesquisadores em torno da solução dos problemas mundiais de deficiência dessa vitamina. Já em 1985, durante a décima reunião do IVACG, em Hyderabad, Índia, pesquisadores brasileiros apresentaram diversos aspectos do programa de suplementação de vitamina A nas campanhas de vacinação no Brasil, à época circunscrito ao estado da Paraíba ${ }^{33}$. 
Nos anos seguintes, diversos países e pilados pela OMS demonstravam que 40 países organizações internacionais, como a OMS e 0 haviam adotado a distribuição de vitamina A nos UNICEF, se interessaram por essa estratégia ${ }^{26}$, que dias nacionais de vacinação anti-pólio, com foi rapidamente difundida, com a colaboração de pesquisadores brasileiros. Em 1998, dados comcoberturas variando entre $30 \%$ e $100 \%$ (Quadro 1). Segundo esses dados, mais de 60 milhões de

Quadro 1. Resultados da suplementação com vitamina A durante campanhas nacionais de imunização anti-poliomielite, diversos países, 1998.

\begin{tabular}{|c|c|c|c|c|}
\hline \multirow[b]{2}{*}{ País } & \multicolumn{2}{|c|}{ População } & \multicolumn{2}{|c|}{ Cobertura } \\
\hline & $\begin{array}{c}\text { Alvo para receber } \\
\text { vitamina } A\end{array}$ & $\begin{array}{c}\text { Suplementada com } \\
\text { vitamina A }\end{array}$ & $\begin{array}{c}\text { Suplementação com } \\
\text { vitamina A (\%) }\end{array}$ & $\begin{array}{l}\text { Vacinação contra } \\
\text { poliomielite (\%) }\end{array}$ \\
\hline 1. Afeganistão & 4.188 .758 & 1.470 .000 & 35 & 76 \\
\hline 2. Bangladesh & 17.914 .845 & 14.331 .876 & 80 & 100 \\
\hline 3. Benin & 890.737 & 1.214 .224 & 136 & 116 \\
\hline 4. Bolívia & 1.073 .264 & 476.302 & 44 & NA \\
\hline 5. Brasil & 4.395 .564 & 1.864 .379 & 42 & NA \\
\hline 6. Burkina Fasso & 1.882 .496 & 1.831 .571 & 97 & 101 \\
\hline 7. Camboja & 1.494 .444 & 1.366 .587 & 97 & 98 \\
\hline 8. Camarões & 597.324 & 776.250 & 130 & 115 \\
\hline 9. Chade & 979.200 & 930.240 & 95 & 87 \\
\hline 10. Coréia & 1.879 .675 & 1.033 .812 & 55 & 100 \\
\hline 11. República Dominicana & 939.095 & 278.902 & 30 & NA \\
\hline 12. Equador & 853.211 & 408.456 & 48 & NA \\
\hline 13. Guiné Equatorial & NA & NA & NA & NA \\
\hline 14. Eritreia & 310.700 & NA & NA & 87 \\
\hline 15. Etiópia & 8.186 .220 & 6.772 .303 & 83 & 107 \\
\hline 16. Gana & 3.721 .884 & 3.457 .049 & 93 & 104 \\
\hline 17. Guatemala & 1.021 .186 & 405.063 & 40 & NA \\
\hline 18. Guiné & 1.438 .633 & 1.348 .237 & 94 & 99 \\
\hline 19. Honduras & NA & NA & NA & NA \\
\hline 20. Irã & NA & NA & NA & NA \\
\hline 21. Kiribati & NA & NA & NA & NA \\
\hline 22. Laos & 618.391 & 554.849 & 90 & 89 \\
\hline 23. Lesoto & NA & NA & NA & NA \\
\hline 24. Madagascar & 2.321 .392 & 2.460 .675 & 106 & 112 \\
\hline 25. Mali & 1.885 .625 & 2.055 .336 & 109 & 113 \\
\hline 26. Mauritânia & 396.392 & 301.258 & 76 & 92 \\
\hline 27. Marrocos & 750.000 & 334.110 & 45 & 95 \\
\hline 28. Namíbia & 279.668 & 246.108 & 88 & 86 \\
\hline 29. Nepal & 831.830 & 748.647 & 90 & 96 \\
\hline 30. Nicarágua & 716.697 & 446.433 & 62 & NA \\
\hline 31. Níger & 1.781 .839 & 1.924 .386 & 108 & 107 \\
\hline 32. Filipinas & 2.148 .214 & 1.958 .234 & 81 & 96 \\
\hline 33. Ruanda & 1.103 .733 & 960.248 & 87 & 89 \\
\hline 34. Somália & 898.842 & 907.106 & 101 & 118 \\
\hline 35. Suazilândia & 154.978 & 120.386 & 78 & NA \\
\hline 36. Togo & 229.026 & 241.644 & 106 & 109 \\
\hline 37. Uganda & 85.111 & 80.770 & 95 & 107 \\
\hline 38. Vietnam & 4.727 .000 & 4.680 .038 & 99 & 99 \\
\hline 39. lêmen & 3.300 .000 & 3.128 .599 & 94 & 116 \\
\hline 40. Zâmbia & 1.945 .108 & 1.762 .005 & 95 & 94 \\
\hline Total & & 60.876 .083 & & \\
\hline
\end{tabular}

Fonte: Goodman et al. ${ }^{34}$. 
crianças foram beneficiadas com suplementos de vitamina A em 1998. A OM S considera que existe ainda um longo caminho a percorrer e algumas janelas de oportunidade a utilizar, tendo em vista que a hipovitaminose A pode ser considerada como um problema de Saúde Pública em 118 países, dos quais 89 (75\%) desenvolvem campanhas de vacinação. Os dados do Quadro 1, contudo, demonstram que menos da metade deles (apenas 40) fazem a associação da vitamina $A$ à vacinação ${ }^{34}$.

Comparativamente ao panorama internacional, as coberturas registradas no Brasil entre 1994 e 2003 , de $28 \%$ a $72 \%$, encontravam-se em patamares semelhantes àqueles de outros países que empregam essa estratégia, como se pode apreender dos dados apresentados no Quadro 1.

As vantagens e os problemas encontrados em outros países também guardam semelhança com os aqui relatados. A OMS destaca, entre os fatores positivos, os relacionados à satisfação das famílias em receber a dose de vitamina, que, em muitos casos, tem contribuído para aumentar 0 comparecimento das crianças aos postos de vacinação. Existe também um grande interesse por parte dos gestores de saúde, pela oportunidade de implementar simultaneamente, a um custo reduzido, duas medidas de saúde pública que, comprovadamente, reduzem a mortalidade infantil ${ }^{34}$. Contudo, há que levar em conta que a logística de distribuição de vitamina A implica em um número maior de profissionais treinados trabalhando nos dias de vacinação, na modificação da rotina e do fluxo de atendimento nos postos, em novos materiais e formulários de controle a serem providenciados, etc. Num balanço geral, a integração da vitamina A aos dias nacionais de vacinação tem mais pontos positivos do que negativos ${ }^{34}$.

\section{O N C L U S Ã O}

Nas últimas três décadas, o quadro epidemiológico da carência de vitamina A no Brasil vem demonstrando a necessidade de intervenções eficazes, para reduzir a prevalência elevada dessa deficiência. Porém, as informações disponíveis não são suficientes para que se possa diagnosticar a magnitude e a gravidade da deficiência dessa vitamina no País como um todo. Considerandose, ademais, o efeito protetor que a suplementação deste micronutriente exerce sobre a saúde infantil, a suplementação com a vitamina A foi implantada em regiões consideradas endêmicas para esta carência, como uma medida de intervenção em curto prazo, para reduzir a mortalidade e prevenir a cegueira nutricional. Contudo, outras ações mais amplas, especialmente aquelas que garantam a diversificação dietética e 0 aporte adequado desta vitamina, são de fundamental importância para o controle da deficiência da vitamina A na população.

Desde 1983, o M inistério da Saúde distribui cápsulas de vitamina $A$, entretanto, o Programa de Controle da Deficiência da Vitamina nunca foi adequadamente avaliado, sendo escassa a documentação sobre as fases de implantação, implementação e consolidação dele. Não existe no País, até a data atual, um sistema nacional de vigilância epidemiológica sobre a deficiência da vitamina $A$, logo, não é possível avaliar o impacto das medidas de prevenção a partir do Programa de Combate à Deficiência dessa Vitamina. Certamente, a inexistência de dados sobre a prevalência do problema no País ou nas macroregiões, teve impacto negativo no alcance do sucesso da implantação desse Programa.

Em termos de cobertura populacional, observa-se que foram modestos os avanços obtidos até 0 ano de 1992, fato que pode ser explicado pela irregularidade na distribuição das cápsulas de vitamina A. A partir de 1994, o programa manteve certa constância, talvez em decorrência de uma base legal gerada pela da Portaria n.2.160, de 29 de Dezembro de 1994, porém persiste a necessidade de promover ações mais consolidadas nos serviços de saúde que atinjam maior alcance social, para além da distribuição rotineira dessas cápsulas. 


\section{REFERÊ N CIA S}

1. Underw ood BA. Maternal vitamin A status and its importance in infancy and early childhood. Am J Clin Nutr. 1994; 59(2 Suppl):517-24.

2. World Health Organization. Indicators for assessing vitamin A deficiency and their application in monitoring and evaluating intervention programmes. M icronutrient series. Geneva: WHO; 1996.

3. United Nations. The world nutrition situation. Nutrition throughout the life cycle. 4th. Geneva: WHO; 2000.

4. World Health Organization. Vitamin A supplementation. Report of an informal consultation, 1-3 March 2000 Yverdon-les-Bains, Switzerland. Geneva: WHO; 2001.

5. Organización Panamericana de la Salud. Visión integrada de la suplementación com vitamina A en las Américas. Informe de la Reunión Regional. Managua. Nicaragua; OPAS; 2001.

6. Instituto Brasileiro de Geografia e Estatística. Perfil estatístico de crianças e mães no Brasil: aspectos nutricionais, 1974-75. Rio de Janeiro: IBGE; 1982.

7. Batista-Filho M, Torres MAA. Acesso à terra e situação nutricional em populações do semi-árido nordestino. Rev Pernambucana Desenv. 1982; 9(1):101-19.

8. Batista-Filho M. Nutrição, alimentação e agricultura no Nordeste Brasileiro: subsídios para uma política de extensão rural. 2a. ed. Brasília: FAO; 1987.

9. M cAuliffe J, Santos LM P, Diniz AS, Batista-Filho M, Barbosa RCC. A deficiência de vitamina A e estratégias para o seu controle: um guia para as Secretarias M unicipais de Saúde. Fortaleza: Project HOPE; 1991.

10. Mariath JGR, Lima MCC, Santos LMP. Vitamin A activity of buriti (Mauritia vinifera $M$ art) and its effectiveness in the treatment and prevention of xerophthalmia. Am J Clin Nutr. 1989; 49(5): 849-53.

11. Santos LM P, Dricot JM, Asciutti LS, Dricot-d'Ans C. Xerophthalmia in the state of Paraíba, Northeast Brazil: clinical findings. Am J Clin Nutr. 1983; 38(1):139-44.

12. Dricot-d'Ans C, Dricot JM, Diniz AS, M ariath JGR, Santos LMP. Geographic distribution of xerophthalmia in the state of Paraiba, Northeast Brazil. Ecology Food Nutr. 1988; 22 (2):131-38.

13. Santos LM P, Assis AMO, M artins M C, Araújo MPN, Morris SS, Barreto ML. Situação nutricional e alimentar de pré-escolares no semi-árido da Bahia (Brasil): II - hipovitaminose A. Rev Saúde Pública. 1996; 30(1):67-74.
14. Araújo RL. Situação alimentar e nutricional do Brasil. Brasília: Tipogresso; 1989.

15. Diniz AS. Aspectos clínicos, subclínicos e epidemiológicos da hipovitaminose A no Estado da Paraíba [tese]. Recife: Universidade Federal de Pernambuco; 1997.

16. Brasil. M inistério da Saúde. II Pesquisa Estadual de Saúde e Nutrição; saúde, nutrição, alimentação e condições sócio-econômicas no Estado de Pernambuco. Recife: Instituto Materno e Infantil de Pernambuco; 1998.

17. Martins MC, Santos LMP, Assis AM O. Prevalência da hipovitaminose a em pré-escolares no Estado de Sergipe. Rev Saúde Pública. 2004; 38(4): 537-42.

18. Marinho HA. Prevalência da deficiência de vitamina A em pré-escolares de três capitais da Amazônia Ocidental brasileira [tese]. São Paulo: Universidade de São Paulo; 1997.

19. Beaton GH, Martorell R, L'Abbe KA, Edmonston B, McCabe G, Rossi AC, et al. Effectiveness of vitamin A supplementation in the control of young child morbidity and Mortality in developing countries. State-of-the-art series, nutrition policy discussion paper n 13. Geneva: WHO; 1993.

20. Barreto ML, Santos LM P, Assis AM O, Araújo M PN, Farenzena GG, Santos PA. Effect of vitamin A supplementation on diarrhea and acute lower-respiratory tract infection in young children in Brazil. Lancet. 1994; 334(8917):228-31.

21. Donabedian A. The role of outcomes in quality assessment and assurance. Quality Rev Bull. 1992; 18(11):356-60.

22. Silva LM V, Formigli VL. A avaliação em saúde: limites e perspectivas. Cad Saúde Pública. 1994; 10(1): 80-91.

23. Contandriopoulos AP, Champagne F, Denis JF, Pineault R. A avaliação na área da saúde: conceitos e métodos. In: Hartz ZM A, organizador. Avaliação em Saúde. Rio de Janeiro: Editora Fiocruz; 1997. p.29-48.

24. Santos SMS, Santos LMP. Methodological approach to a multidimensional evaluation of food and nutrition policies. Forum of Nutrition; M odern Aspects of Nutrition. 2003; 56:115-8.

25. Lobato A. Programa e projetos para o controle da hipovitaminose A no Brasil. Relatório elaborado para a FAO. 1988 [M imeografado].

26. World Health Organization. International Vitamin A Consultative Group. Using Immunization Contacts to Combat Vitamin A Deficiency. Report of an Informal Consultative Group of the World Health Organization. Geneva: WHO; 1993. 
27. Brasil. Portaria no 2.160 , de 23 de dezembro de 1994. Cria, no Instituto Nacional de Alimentação e Nutrição, o Programa Nacional de Controle da Deficiência de Vitamina A, e dá outras providências. Brasília: Diário Oficial da República Federativa da Brasil, n. 248, 30 dez. 1994 [acesso em 23 jan. 2007]. Disponível em: http://portalweb01.saude. gov.br/alimentacao/documentos/portaria2160.pdf

28. Brasil. Portaria no 729, de 13 de maio de 2005. Institui o Programa Nacional de Suplementação de Vitamina A [acesso em 23 jan 2007]. Disponível em: http://dtr2004.saude.gov.br/nutricao/ documentos/vita/portaria_729_vita.pdf

29. Santos LM .P. Que falta faz a vitamina A? Ciência Hoje. 1987; 4(23):8-9.

30. Assis AM O, Santos LM P, Prado MS, Martins MC, Barreto ML. B. Tolerância à aplicação de megadoses de vitamina $A$ associada à vacinação em crianças no Nordeste do Brasil. Cad Saúde Pública. 2000; 16(1):51-7.

31. Martins MC, Santos LMP. Avaliação do Programa Nacional de Controle da Deficiência de Vitamina
A na Bahia. In: Santos SM C, Santos LM P. Avaliação de Políticas Públicas de Segurança Alimentar e Nutrição no estado da Bahia. Relatório final apresentado à FINEP. Salvador: Bureau Editora; 2004.

32. Brasil. Ministério da Saúde. Coordenação Geral da Política de Alimentação e Nutrição. Alimentos Regionais Brasileiros. Brasília: Ministério da Saúde; 2002.

33. Santos LM P, D’Ans CD, M ariath JGR, Dricot J, Diniz, AS. A preventive program for vitamin A deficiency in Paraíba, Northeast Brazil. Annals of the $X$ International Vitamin. Consultative Group Meeting [Abstract]. Hyderabad, India: IVACG; 1985.

34. Goodman T, Dalmya N, Benoist B, Schultink W. Polio as platform: using national immunization days to deliver vitamin A supplements. Bull World Health Org. 2000; 78(3):305-14.

Recebido em: 30/9/2005

Versão final reapresentada em: 6/11/2006 Aprovado em: 21/12/2006 